\title{
Vascular index measured by smart 3-D superb microvascular imaging can help to differentiate malignant and benign breast lesion
}

This article was published in the following Dove Press journal:

Cancer Management and Research

\author{
Xiao-Yan Zhang' \\ Li Zhang' \\ $\mathrm{Na} \mathrm{Li}{ }^{l}$ \\ Qing-Li Zhu' \\ Jian-Chu Li ${ }^{\text {I }}$ \\ Qiang Sun ${ }^{2}$ \\ Hong-Yan Wang ${ }^{1, *}$ \\ Yu-Xin Jiang ${ }^{\mathrm{l} *}$ \\ 'Department of Diagnostic Ultrasound, \\ Chinese Academy of Medical Sciences \\ and Peking Union Medical College \\ Hospital, Beijing 100730, People's \\ Republic of China; ${ }^{2}$ Department of Breast \\ Surgery, Chinese Academy of Medical \\ Sciences and Peking Union Medical \\ College Hospital, Beijing 100730, People's \\ Republic of China
}

*These authors contributed equally to this work
Correspondence: Hong-Yan Wang; Yu-Xin Jiang

Department of Diagnostic Ultrasound, Chinese Academy Medical Sciences and Peking Union Medical College Hospital, No.I Shuai Fu Yuan, Dong Cheng District, Beijing 100730, People's Republic of China $\mathrm{Tel}+86106915$ 5494; +86 1069155491 Fax +86 I06 915 5494; +86 1069155402 Email whychina@I26.com; jiangyuxinxh@163.com
Purpose: The purpose of our study was to prospectively evaluate the diagnostic performance of the vascular index (VI, defined as the ratio of Doppler signal pixels to pixels in the total lesion) measured via Smart 3-D superb microvascular imaging (SMI) for breast lesions. Patients and methods: Two hundred and thirty-two consecutive patients with 236 breast lesions referred for biopsy at Peking Union Medical College Hospital were enrolled in the study from December 2016 to November 2017. Sensitivity, specificity, positive predictive value (PPV), negative predictive value (NPV) and accuracy of VI were calculated with histopathologic results as the reference standard.

Results: Of the 236 breast lesions, 121 were malignant and 115 were benign. The mean VI was significantly higher in malignant lesions $(9.7 \pm 8.2)$ than that in benign ones $(3.4 \pm 3.3)$ $(P<0.0001)$. Sensitivity, specificity, PPV, NPV and accuracy of VI (4.0 as the threshold) were respectively: $76.0 \%, 66.1 \%, 70.2 \%, 72.4 \%$ and $71.2 \%(P<0.05)$.

Conclusion: Smart three-dimensional (3-D) SMI is a noninvasive tool using twodimensional (2-D) scanning to generate 3-D vascular architecture with a high-resolution image of micro-vessels. This can be used as a qualitative guide to identify the optimal 2-D SMI plane with the most abundant vasculature to guide VI quantitative measurements of breast lesions. Smart 3-D SMI may potentially serve as a noninvasive tool to accurately characterize benign versus malignant breast lesions.

Keywords: breast neoplasms, ultrasonography, diagnostic imaging, superb microvascular imaging

\section{Introduction}

Angiogenesis plays a crucial role in the progression of tumor growth and metastasis. ${ }^{1,2}$ Malignant tumors tend to show increased vascularization. Therefore, a variety of imaging modalities were used to analyze tumor-associated vasculature.

Color or power Doppler ultrasound (US) is widely applied to evaluate tumor angiogenesis in breast cancer. Feeding vessels are generated to sustain the growth of cancer; high-grade high-cellular tumors usually have abundant vasculature, while low-grade tumors or tumors with central necrosis may have no demonstrable vascularization on Doppler US. $^{3}$ In addition, the fine vessels of breast lesions may not be detected by color or power Doppler US because of the low velocity and artifacts. ${ }^{4-6}$ Contrast-enhanced MRI could identify breast tumor properties, but MRI contrast agents do not remain entirely within the intravascular space; rather, a considerable portion is distributed into the surrounding extracellular spaces, 
which may interfere with the evaluation of the vasculature. $^{7}$ In addition, contrast-enhanced MRI is contraindicated in certain patients, including those with pacemakers, renal impairment and others. With the advent of US contrast agents, several clinical studies have reported promising results using contrast-enhanced ultrasound (CEUS) in the breast cancer diagnosis. ${ }^{8-10}$ CEUS can identify slow and low-volume blood flow inside tumors from 20 to $39 \mu \mathrm{m}$ in diameter; ${ }^{6}$ US contrast agents remain solely intravascular, enabling direct evaluation of the vasculature; however, CEUS is an imaging modality that requires an interventional procedure, injection of a contrast medium.

Superb microvascular imaging (SMI) technology is an innovative Doppler vascular imaging technique, which uses an adaptive algorithm to highlight subtle and dismiss flow signals from tissue motion artifacts. Blood flow and motion artifacts (clutters) are simultaneously produced by US imaging. The new adaptive algorithm isolates and removes clutters while preserving the underlying hemodynamic flow information. SMI mode can visualize lowvelocity flow with excellent resolution, outstanding frame rate and minimal flash artifacts. Low-speed flow is displayed more clearly with SMI in detail than color or power Doppler imaging. ${ }^{11,12}$ Furthermore, SMI is a noninvasive modality with no contraindications. It does not require an injection of a contrast agent or MRI scanning. Initial experiences of SMI in breast lesions have suggested that SMI is more sensitive to blood flow signals in breast lesions than color Doppler or power Doppler US. ${ }^{13,14}$ This modality also displays more penetrating vessels in avascular breast lesions compared to color Doppler imaging. ${ }^{15}$ Several similar techniques such as micro-flow imaging (MFI) and Micro $\mathrm{V}$ had been reported. ${ }^{16}$ The noncontrast MFI and micro V are the latest technologies, which employ an advanced algorithm and can efficiently distinguish the Doppler signals derived from tissue movement and blood flow; they are capable of detecting very small vessels and slow flows without the use of contrast agent. However, SMI, MFI and Micro V are semiquantitative methods to evaluate the richness of the blood flow. They cannot be used to quantitatively assess the vasculature.

The recently introduced Smart 3-D SMI can reconstruct three-dimensional (3-D) images from twodimensional (2-D) images scanning with a linear array probe. It can be used to visualize the blood flow pattern of low-velocity flow in 3-D mode and quantitatively assess tumor vascularity via measuring vascularity index (VI) on 2-D SMI images obtained with the qualitative guidance of 3-D SMI images. To our knowledge, there have been no reports on VI measured using Smart 3-D SMI technology to assess breast vasculature to differentiate breast lesions. The aim of our study was to evaluate the diagnostic performance of the VI measured via Smart 3-D SMI for breast lesions.

\section{Material and methods \\ Patients}

The Ethics Committee of Peking Union Medical College Hospital approved the prospective study, and all patients provided written informed consent for the study. From December 2016 to November 2017, 232 consecutive female patients (mean age $44.9 \pm 12.5$ years, range 19-65 years,) with 236 breast lesions on US were included in the study. The reasons of patients' seeking medical care included (1) self-discovered breast mass; (2) breast masses by US screening or mammography; (3) nipple discharge with breast masses on US; (4) chest wall mass recurrence after breast cancer surgery. The exclusion criteria were as follows: (1) lesions larger than the probe, because the US parameter would be shielded. (2) Patients who had undergone a previous breast biopsy or surgery, because minimal vessel injury may have altered the blood supply to the targeted lesion. (3)Pregnant women were excluded because breast parenchymal changes can also alter the blood supply to the targeted lesion. All patients underwent excision biopsy and were histopathologically examined.

\section{Smart 3-D SMI}

All lesions were detected using the US Aplio 500 (L14-5, Aplio 500; Toshiba Medical Systems, Tokyo, Japan) which can perform Smart 3-D examination. SMI examination was performed using color mode. The parameters of the Smart 3-D SMI were set to a low-velocity range $(1.2-1.6 \mathrm{~cm} / \mathrm{s})$ to visualize extremely low-velocity flow with good resolution and a high frame rate with minimal flash artifacts (frame rate, 25-30/s; pulse repetition frequency, 15.4-20.2 kHz). The range of depth was adjusted to $2.5-4 \mathrm{~cm}$ according to lesion size, and the detectable width of the linear probe was $6 \mathrm{~cm}$. Smart 3-D reconstructs 3-D volumes from 2-D SMI images using conventional 2-D transducers. 3-D SMI volumes were acquired by scanning with a linear US transducer, without the need of a 3-D transducer. With a one-button 
action, the 3-D SMI images were immediately reconstructed to create an entire, 3-D vasculature volume with high resolution of the tissue vasculature with clear visualization of branching vessels.

3-D SMI was used as a qualitative guidance to identify the 2-D SMI plane with the most abundant vasculature, and VI value was achieved by manually tracing the boundary of the lesion on the 2-D SMI image by a radiologist for three times and averaged. One radiologist (HW with $>15$ years of ultrasonic work experience and 2 months of experience in SMI) conducted US and Smart 3-D SMI examinations. The total inspection time was about 20 mins.

\section{Statistical analysis}

Continuous variables were expressed by mean \pm SD. Student's $t$-test was used to compare the difference of quantitative data and chi-square test for count data. Sensitivity, specificity, positive predictive value (PPV), negative predictive value (NPV) and accuracy of VI were calculated using receiver operating characteristics (ROC) curve with histopathologic diagnosis as the reference standard. Youden index was used to determine the threshold of VI in diagnosing benign and malignant breast lesions. Area under ROC curves were calculated. Twotailed $P$-values less than 0.05 were statistically significant. SPSS 18.0 software (version 20; SPSS Inc., Chicago, IL, USA) was used for all statistic analysis.

\section{Results}

All 236 lesions were confirmed by pathology; 121 lesions in 119 women (mean age 51.6 \pm 12.8 years, range $23-83$ years) were malignant and 115 lesions in 113 women (mean age $41.5 \pm 12.0$ years, range $19-78$ years) were benign (Table 1). The mean age of patients in the malignant group were older than the benign group ( $\mathrm{t}=-6.275$, $P<0.001)$. Average size of the malignant lesions ( 2.8 $\pm 1.8 \mathrm{~cm}$, range $0.5-11.7 \mathrm{~cm}$ ) was significantly larger than the benign lesions $(1.8 \pm 1.2 \mathrm{~cm}$, range $0.3-7.9 \mathrm{~cm})$ $(\mathrm{t}=-5.300, P<0.001)$. The VI distributions for the 236 malignant and benign lesions are shown in Figure 1. The mean VI was significantly higher in malignant (mean VI $9.7 \pm 8.2$, range $0-48$ ) versus benign breast lesions (mean VI 3.4 \pm 3.3 , range $0-15.9)(\mathrm{t}=-7.810, P<0.001)$ (Figure 2).

ROC curve was applied to identify the maximum value of sensitivity and specificity. Using a VI of 4.0 (Table 2) as the threshold value to identify malignant lesions from benign lesions yielded the sensitivity $76.0 \%$, the
Table I Histopathologic diagnosis of 236 breast lesions

\begin{tabular}{|l|l|}
\hline Histopathologic diagnosis & N (\%) \\
\hline Benign lesions & \\
fibroadenoma & $52(22.0)$ \\
Mammary adenosis & $29(12.3)$ \\
Intraductal papilloma & $20(8.5)$ \\
Mastitis & $8(3.3)$ \\
Scar granuloma & $6(2.5)$ \\
\hline Malignant lesions & \\
Infiltrating ductal carcinoma & $87(36.9)$ \\
Ductal carcinoma in situ & $18(7.6)$ \\
Infiltrating lobular carcinoma & $3(1.3)$ \\
Mucous carcinoma & $3(1.3)$ \\
Solid papillary carcinoma & $2(0.9)$ \\
Sarcoma & $2(0.9)$ \\
Borderline/malignant phyllodes tumor & $2(0.9)$ \\
Infiltrating tubular carcinoma & $\mathrm{I}(0.4)$ \\
Invasive tubular carcinoma & $\mathrm{I}(0.4)$ \\
Neuroendocrine carcinoma & $\mathrm{I}(0.4)$ \\
Paget's desease & $\mathrm{I}(0.4)$ \\
\hline Total & $236(100)$ \\
\hline
\end{tabular}

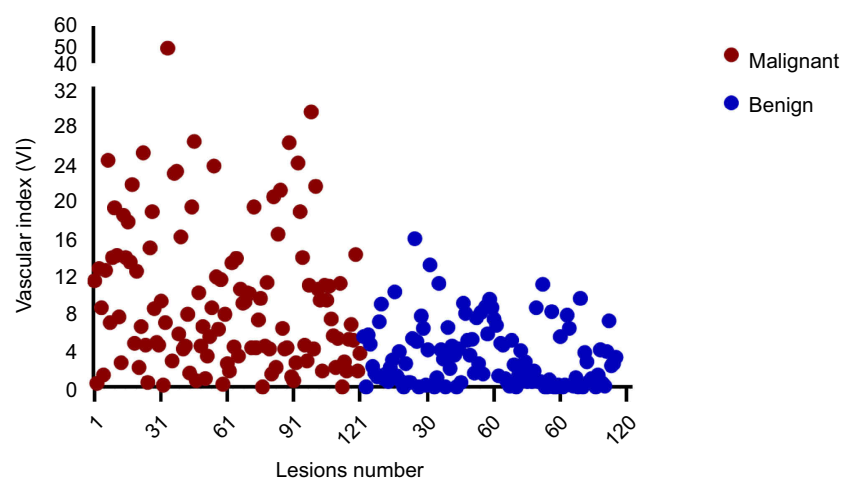

Figure I Scatter plot shows vascular index (VI) distribution in 236 malignant and benign lesions. Patients' codes are shown on the horizontal axis. Red dots represent malignant lesions and blue dots benign ones.

specificity $66.1 \%$, the PPV $70.2 \%$, the NPV $72.4 \%$ and the AUC $0.776(0.72 \sim 0.83)$. The first to third quartile interval of VI for benign breast lesions was 4.9 (range 0.5-5.4) and 9.8 (range 4.1-13.9) for malignant ones.

Among all the lesions, there were $39(33.9 \%, 39 / 115)$ benign lesions with VI higher than 4.0 and $29(24.0 \%, 29 /$ 121) malignant lesions with VI less than 4.0 (Table 3 ). The most common pathologic types of false positives with VI were fibroadenoma $(14 / 39,36 \%)$; intraductal papilloma (2/ $39,31 \%)$ and adenosis $(8 / 39,21 \%)$; the most common pathologic type of false negatives with VI was infiltrating ductal carcinoma $(16 / 29,55 \%)$ and the subtypes were 10 


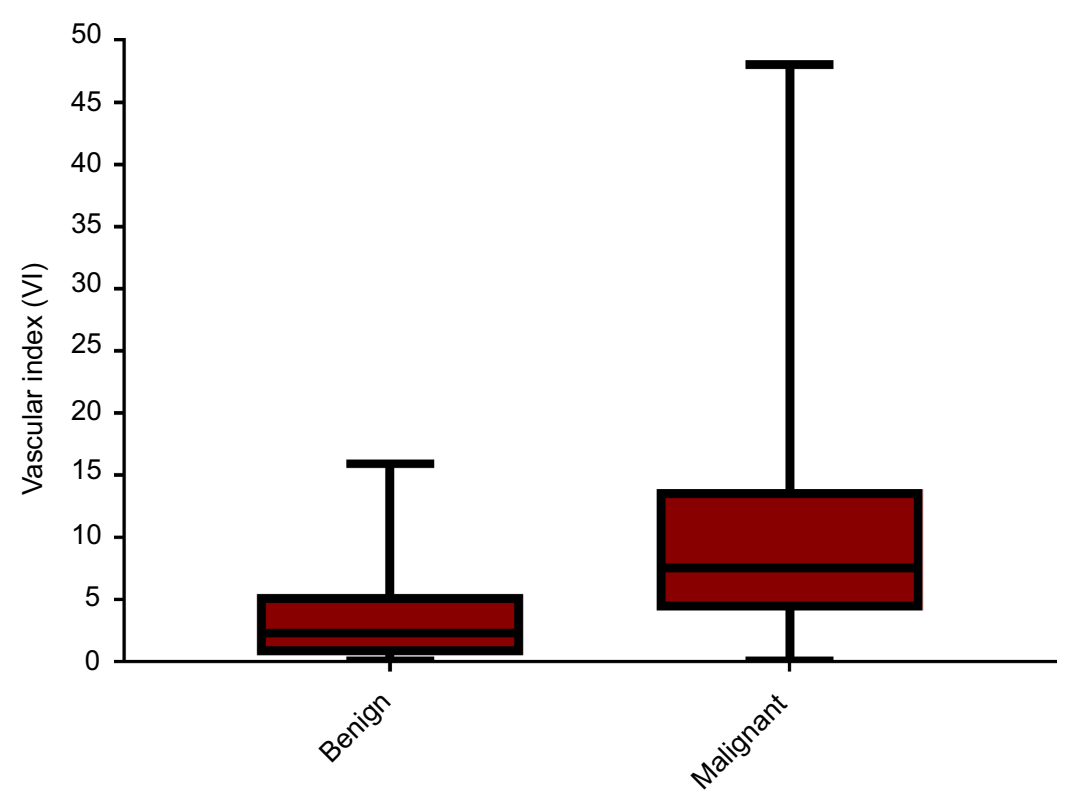

Pathology

Figure 2 Box plot of vascular index distribution in 236 breast lesions. The mean vascular index (VI) was significantly higher in patients with malignant breast lesions (mean $\mathrm{VI}$ 9.7 \pm 8.2 , range $0-48$ ) than that in patients with benign breast lesions (mean $\mathrm{VI} 3.4 \pm 3.3$, range $0-15.9)(P<0.0001)$.

Table 2 Sensitivity and specificity of $\mathrm{VI}$ at various cutoff points for the diagnosis of breast lesions

\begin{tabular}{|l|l|l|l|}
\hline Cutoff of VI & Sensitivity & Specificity & Youden index \\
\hline 3.5000 & 0.769 & 0.600 & 0.369 \\
3.9500 & 0.760 & 0.634 & 0.395 \\
4.0500 & 0.760 & 0.661 & 0.421 \\
4.1500 & 0.727 & 0.669 & 0.396 \\
4.2500 & 0.702 & 0.669 & 0.372 \\
\hline
\end{tabular}

Luminal B (HER2-positive), three HER2-positive, two Luminal A and one triple-negative.

\section{Discussion}

Breast cancer is the major cause of cancer death among females worldwide. ${ }^{17}$ US is a convenient procedure for examining breast lesions, particularly those in dense breasts, and is a suitable modality for repeated examinations. Gray-scale

Table 3 Characteristics of false-positive and false-negative lesions with VI measured via Smart 3-D SMI

\begin{tabular}{|l|l|l|l|}
\hline Pathologic diagnosis & N(\%) & Size (cm) & Mean VI \\
\hline False positive & & & $7.0 \pm 1.9$ \\
$\quad$ Fibroadenoma & $14(35.8)$ & $1.7 \pm 0.5$ & $7.8 \pm 3.6$ \\
Intraductal papilloma & $12(30.8)$ & $1.6 \pm 2.1$ & $7.2 \pm 2.2$ \\
Mammary adenosis & $8(20.5)$ & $1.8 \pm 0.6$ & $7.1 \pm 2.5$ \\
Mastitis & $4(10.3)$ & $1.9 \pm 1.0$ & 8.5 \\
Scar granuloma & $\mathrm{I}(2.6)$ & 1 & $7.4 \pm 2.6$ \\
Total & $39(100)$ & $1.7 \pm 1.3$ & \\
\hline False negative & & & $2.0 \pm 0.9$ \\
Infiltrating ductal carcinoma & $16(55.2)$ & $2.5 \pm 1.5$ & $2.1 \pm 1.0$ \\
Ductal carcinoma in situ & $6(20.7)$ & $4.0 \pm 1.7$ & $0.1 \pm 0.1$ \\
Papillary carcinoma & $2(6.9)$ & $3.4 \pm 3.7$ & $3.1 \pm 0.1$ \\
Sarcoma & $2(6.9)$ & $2.8 \pm 0.6$ & $0.9 \pm 0.3$ \\
Mucous carcinoma & $2(6.9)$ & 5.5 & $0.5 \pm 0.3$ \\
Infiltrating lobula carcinoma & $\mathrm{I}(3.4)$ & $3.1 \pm 1.7$ & 0.5 \\
Total & $29(100)$ & $1.7 \pm 1.1$ \\
\hline
\end{tabular}


ultrasonic characteristics are described in detail in the Breast Imaging Reporting and Data System lexicon, which is an efficient scoring system that is widely used for evaluating breast lesions, whereas fewer angiogenesis characteristics are included in the lexicon. Tumor angiogenesis is necessary for its growth and metastasis. ${ }^{18}$ Tumors usually have abundant vascularity. ${ }^{19}$ Since malignant breast lesions have a higher microvessel density than benign lesions, it is important to interrogate lesion vascularity. Several studies demonstrated that SMI had advantages in showing more tiny vessels in breast carcinoma than color and power Doppler imaging. ${ }^{13,20,21}$ Since SMI can more sensitively detect minimal low-velocity flow qualitative signals in breast lesions, the advancement of Smart 3-D SMI can be used to visualize blood flow pattern and measure VI quantitatively. Our results showing that the mean VI values of malignant breast lesions (Figure 3) significantly exceed those in benign breast lesions (Figure 4) are consistent with previous reports using Doppler US and SMI technology. ${ }^{20,22}$ Using pathology as the reference standard, a cutoff value of 4.0 of VI was applied to reflect the benign versus malignant diagnosis for breast lesions with ideal efficacy.

In our study, the vascular VI values of benign lesions that overlapped with VI values of malignant breast lesions were fibroadenoma, intraductal papilloma and adenosis. These benign breast neoplasms have a rich vascularity with a higher proportion of tumor cells and stroma or adenosis.

Malignant breast tumors are heterogeneous. Some breast carcinomas had relatively little vasculature. Tumor growth is angiogenesis-dependent ${ }^{23}$ and angiogenesis in breast cancer has been reported to be heterogeneous and highly distorted. ${ }^{24}$ Some tumor regions may have an abundant blood supply, while others not. ${ }^{25}$ Boetes et al confirmed that some avascular tumors such as invasive cancers and ductal carcinoma in situ have low blood perfusion on MRI. ${ }^{26}$ Immunohistochemical analysis confirmed a decreased microvessel density at the centers of the malignant breast tumors, which was consistent with the contrastenhanced sonographic findings showing decreased perfusion and even a perfusion defect at the centers. ${ }^{27}$ In our

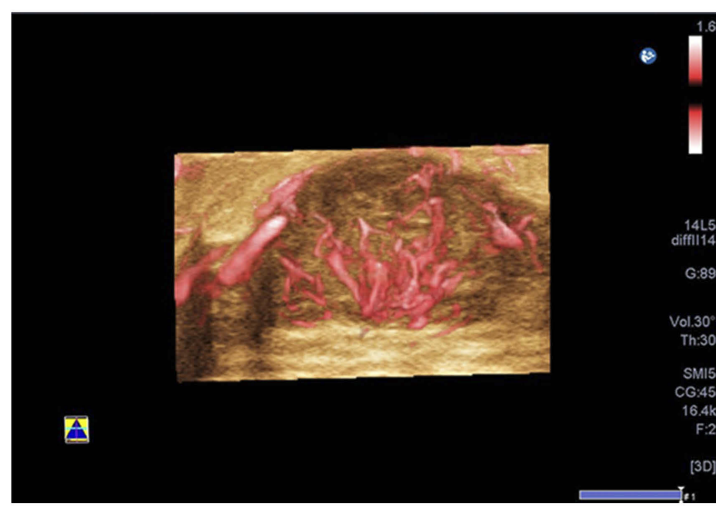

B

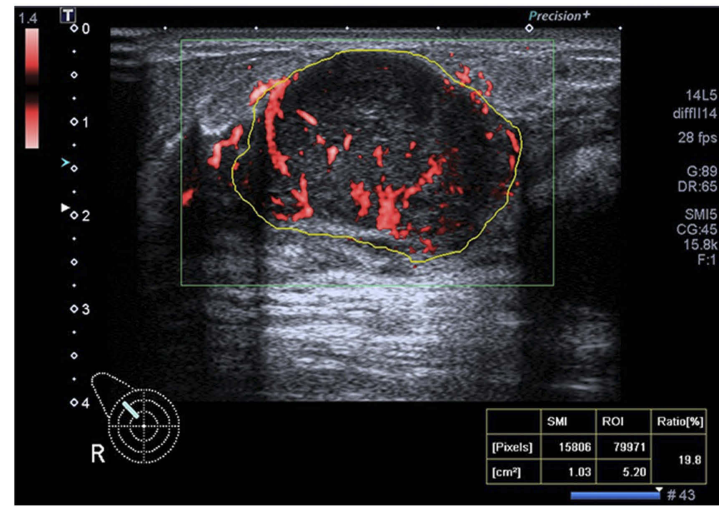

D

Figure 3 A 34-year-old woman with invasive ductal carcinoma. (A) Color Doppler flow image shows abundant and disordered blood flow signals. (B and C) Smart threedimensional superb microvascular imaging reveals detailed and abundant vascular architecture with crab claw-like blood flow. (D) Vascular index was measured on the plane containing the most abundant vasculature with a value of 19.8 . 


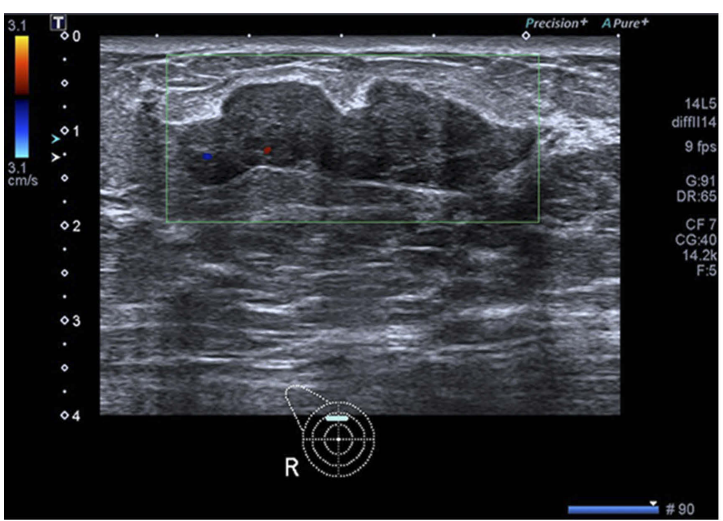

A

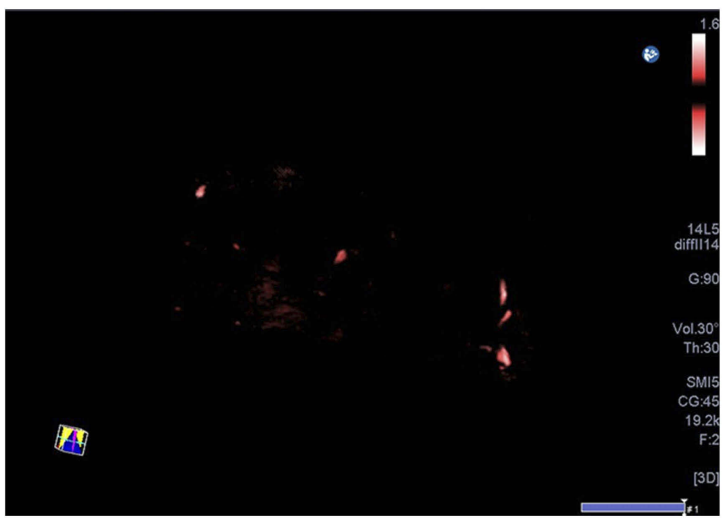

C

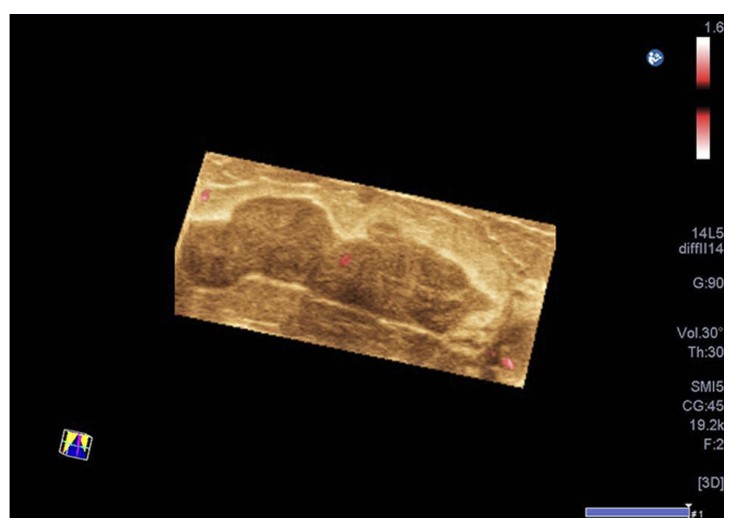

B

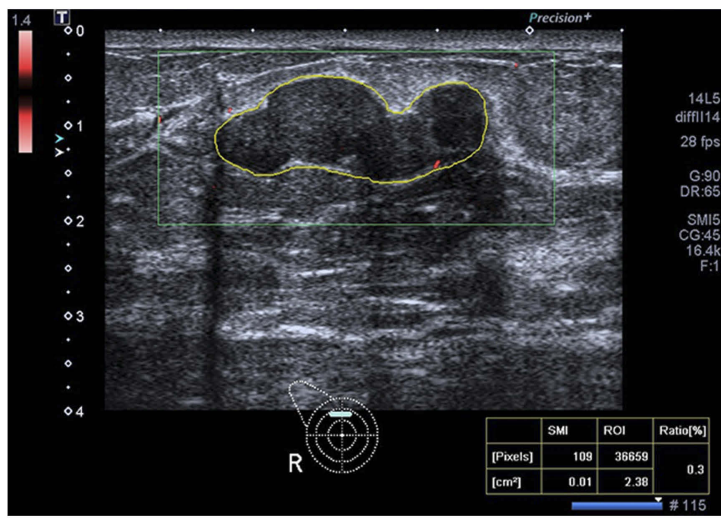

D

Figure 4 A 20-year-old woman with fibroadenoma. (A) Color Doppler flow imaging image shows few blood flow signals. (B and C) Smart three-dimensional superb microvascular imaging reveals few spots and linear blood flow. (D) Vascular index was measured on the plane containing the most abundant vasculature with a value of 0.3 .

study, the VI of malignant breast lesions had a wider distribution interval than that of benign lesions, indicating that angiogenesis can be heterogeneous in malignant tumors. In addition, certain cancers with sclerotic tumor stroma with a hard texture can appear to lack internal vascularity on color Doppler US. ${ }^{28}$

Our study has some limitations. First, 3-D reconstruction based on the acquisition of 2-D data is operator-dependent. The sonographer must maintain a steady speed and pressure during the examination in order to avoid distortion of the Smart 3-D SMI images. Second, this preliminary study evaluated the performance of VI measured by SMI for breast lesions. Comparison of VI with microvessel density count on histology slices could be assessed in further work.

\section{Conclusion}

Smart 3-D SMI is a noninvasive tool using 2-D scanning to generate 3-D vascular architecture with a highresolution image of micro-vessels. This can be used as a qualitative guide to identify the optimal 2-D SMI plane with the most abundant vasculature to guide VI quantitative measurements of breast lesions. Smart 3-D SMI may potentially serve as a noninvasive tool to accurately characterize benign versus malignant breast lesions.

\section{Abbreviation list}

SMI, superb microvascular imaging; US, ultrasound; VI, vascular index; PPV, positive predictive value; NPV, negative predictive value.

\section{Acknowledgment}

This study was supported through funding by the National Natural Science Youth Foundation of China (81601517).

\section{Disclosure}

The authors report no conflicts of interest in this work.

\section{References}

1. Bergers G, Benjamin LE. Tumorigenesis and the angiogenic switch. Nat Rev Cancer. 2003;3(6):401-410. doi:10.1038/nrc1093 
2. Drudi FM, Cantisani V, Gnecchi M, Malpassini F, Di Leo N, de Felice C. Contrast-enhanced ultrasound examination of the breast: a literature review. Ultraschall Med. 2012;33(7):E1-E7. doi:10.1055/s-00311299408

3. Busilacchi P, Draghi F, Preda L, Ferranti C. Has color Doppler a role in the evaluation of mammary lesions? J Ultrasound. 2012;15 (2):93-98. doi:10.1016/j.jus.2012.02.007

4. Kettenbach J, Helbich TH, Huber S, Zuna I, Dock W. Computerassisted quantitative assessment of power Doppler US: effects of microbubble contrast agent in the differentiation of breast tumors. Eur J Radiol. 2005;53(2):238-244. doi:10.1016/j.ejrad.2004.04.017

5. Less JR, Skalak TC, Sevick EM, Jain RK. Microvascular architecture in a mammary carcinoma: branching patterns and vessel dimensions. Cancer Res. 1991;51(1):265-273.

6. Forsberg F, Kuruvilla B, Pascua MB, et al. Comparing contrast-enhanced color flow imaging and pathological measures of breast lesion vascularity. Ultrasound Med Biol. 2008;34 (9):1365-1372. doi:10.1016/j.ultrasmedbio.2008.02.010

7. Teifke A, Behr O, Schmidt M, et al. Dynamic MR imaging of breast lesions: correlation with microvessel distribution pattern and histologic characteristics of prognosis. Radiology. 2006;239(2):351-360. doi:10.1148/radiol.2392050205

8. Faustino-Rocha AI, Gama A, Oliveira PA, et al. A contrast-enhanced ultrasonographic study about the impact of long-term exercise training on mammary tumor vascularization. J Ultrasound Med. 2017. doi:10.1002/jum.14287

9. Saracco A, Szabo BK, Tanczos E, Bergh J, Hatschek T. Contrastenhanced ultrasound (CEUS) in assessing early response among patients with invasive breast cancer undergoing neoadjuvant chemotherapy. Acta Radiol. 2017;58(4):394 402. doi:10.1177/0284185116658322

10. Zhao LX, Liu H, Wei Q, et al. Contrast-enhanced ultrasonography features of breast malignancies with different sizes: correlation with prognostic factors. Biomed Res Int. 2015;2015:613831. doi:10.1155/2015/613831

11. Yongfeng Z, Ping Z, Wengang L, Yang S, Shuangming T. Application of a novel microvascular imaging technique in breast lesion evaluation. Ultrasound Med Biol. 2016;42(9):2097-2105. doi:10.1016/j.ultrasmedbio.2016.05.010

12. Gabriel M, Tomczak J, Snoch-Ziolkiewicz M, Dzieciuchowicz L, Strauss E, Oszkinis G. Comparison of superb micro-vascular ultrasound imaging (SMI) and contrast-enhanced ultrasound (CEUS) for detection of endoleaks after endovascular aneurysm repair (EVAR). Am J Case Rep. 2016;17:43-46.

13. Xiao XY, Chen X, Guan XF, Wu H, Qin W, Luo BM. Superb microvascular imaging in diagnosis of breast lesions: a comparative study with contrast-enhanced ultrasonographic microvascular imaging. $\mathrm{Br}$ J Radiol. 2016;89(1066):20160546. doi:10.1259/bjr.20160546

14. Park AY, Seo BK, Woo OH, et al. The utility of ultrasound superb microvascular imaging for evaluation of breast tumour vascularity: comparison with colour and power Doppler imaging regarding diagnostic performance. Clin Radiol. 2018;73(3):304-311. doi:10.1016/j. crad.2017.10.006
15. Zhan J, Diao XH, Jin JM, Chen L, Chen Y. Superb microvascular imaging - a new vascular detecting ultrasonographic technique for avascular breast masses: a preliminary study. Eur J Radiol. 2016;85 (5):915-921. doi:10.1016/j.ejrad.2015.12.011

16. Han H, Ding H, Ji Z, Zhang W, Wang Q, Wang W. Primary application of micro-flow imaging technology in the diagnosis of hepatic tumors. Ultrasound Med Biol. 2019 ;45(2):395-401. doi:10.1016/j. ultrasmedbio.2018.09.025

17. Torre LA, Bray F, Siegel RL, Ferlay J, Lortet-Tieulent J, Jemal A. Global cancer statistics, 2012. CA Cancer J Clin. 2015;65(2):87-108. doi: $10.3322 /$ caac. 21262

18. Lee WJ, Chu JS, Huang CS, Chang MF, Chang KJ, Chen KM. Breast cancer vascularity: color Doppler sonography and histopathology study. Breast Cancer Res Treat. 1996;37(3):291-298.

19. Gokalp G, Topal U, Kizilkaya E. Power Doppler sonography: anything to add to BI-RADS US in solid breast masses? Eur J Radiol. 2009;70(1):77-85. doi:10.1016/j.ejrad.2007.12.007

20. Ma Y, Li G, Li J, Ren WD. The diagnostic value of superb microvascular imaging (SMI) in detecting blood flow signals of breast lesions: a preliminary study comparing SMI to color doppler flow imaging. Medicine (Baltimore). 2015;94(36):e1502. doi:10.1097/ MD.0000000000000874

21. Park AY, Seo BK, Cha SH, Yeom SK, Lee SW, Chung HH. An innovative ultrasound technique for evaluation of tumor vascularity in breast cancers: superb micro-vascular imaging. J Breast Cancer. 2016;19(2):210-213. doi:10.4048/jbc.2016.19.2.210

22. Lee SW, Choi HY, Baek SY, Lim SM. Role of color and power doppler imaging in differentiating between malignant and benign solid breast masses. J Clin Ultrasound. 2002;30(8):459-464. doi:10.1002/jcu.10100

23. Folkman J. Tumor angiogenesis: therapeutic implications. $N$ Engl $J$ Med. 1971;285(21):1182-1186. doi:10.1056/NEJM197111182 852108

24. Mankoff DA, Dunnwald LK, Gralow JR, et al. Blood flow and metabolism in locally advanced breast cancer: relationship to response to therapy. $J$ Nucl Med. 2002;43(4):500-509.

25. Jain RK. Haemodynamic and transport barriers to the treatment of solid tumours. Int J Radiat Biol. 1991;60(1-2):85-100.

26. Boetes C, Strijk SP, Holland R, Barentsz JO, Van Der Sluis RF, Ruijs JH. False-negative MR imaging of malignant breast tumors. Eur Radiol. 1997;7(8):1231-1234. doi:10.1007/ s003300050281

27. Li YJ, Wen G, Wang Y, et al. Perfusion heterogeneity in breast tumors for assessment of angiogenesis. J Ultrasound Med. 2013;32 (7):1145-1155. doi:10.7863/ultra.32.7.1145

28. Lee SH, Chung J, Choi HY, et al. Evaluation of screening US-detected breast masses by combined use of elastography and color doppler US with B-Mode US in women with dense breasts: a multicenter prospective study. Radiology. 2017;285(2):660-669. doi:10.1148/radiol.2017162424
Cancer Management and Research

\section{Publish your work in this journal}

Cancer Management and Research is an international, peer-reviewed open access journal focusing on cancer research and the optimal use of preventative and integrated treatment interventions to achieve improved outcomes, enhanced survival and quality of life for the cancer patient.
The manuscript management system is completely online and includes a very quick and fair peer-review system, which is all easy to use. Visit http://www.dovepress.com/testimonials.php to read real quotes from published authors. 